\title{
INDUSTRIAL AND ORGANIZATIONAL PSYCHOLOGY
}

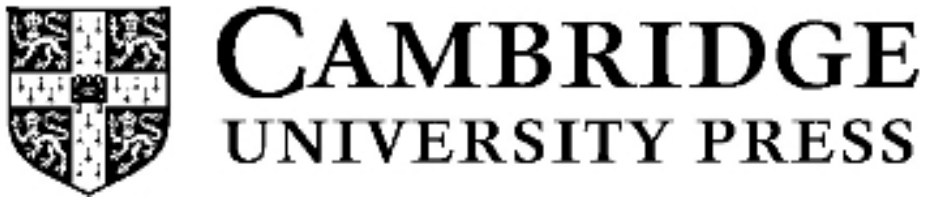

Age bias in the time of coronavirus: Implications for research and practice

\begin{tabular}{|r|l|}
\hline Journal: & Industrial and Organizational Psychology \\
\hline Manuscript ID & IOP-CO-20-133.R2 \\
\hline Manuscript Type: & Commentary \\
\hline Keywords: & Age bias, age diversity, COVID-19, pandemic \\
\hline Abstract: & \\
\hline \multicolumn{2}{|l}{} \\
\end{tabular}

\section{SCHOLARONE ${ }^{m}$ \\ Manuscripts}


In their article, Rudolph et al. (2020) recognized the aging workforce as a key topic relevant to the pandemic for both researchers and practitioners alike, describing a framework of differential susceptibility and differential impact based on lifespan perspectives of aging. We agree that aging workers are of primary importance for psychologists in the pandemic. However, we propose that the differential susceptibility and impact framework can also usefully be considered from a social psychology and age diversity perspective. We argue that the pandemic does not only affect the aging workforce due to natural developmental processes or increased physical susceptibility to illness but, rather, the pandemic exacerbates existing attitudes and biases towards older workers in age diverse teams and organisations, which can lead to a more hostile work environment for older workers, diminishing team effectiveness and limiting older workers' access to work opportunities.

Next, we outline why and how age-based social categorization processes and biases might be affected during the pandemic. We then offer suggestions for research and practice.

\section{Age-Based Social Categorization Processes During the Pandemic}

Social categorization perspectives of diversity suggest that diverse teams tend to form subgroups based on perceived similarities and differences between group members, such as age, gender, or personality (Tajfel \& Turner, 1986; Turner et al., 1987). These processes can lead to intergroup bias, and have been associated with increased interpersonal conflict, and negative performance outcomes (Thatcher \& Patel, 2012). A key feature of this theoretical perspective is categorization salience, which is the extent to which a demographic category is cognitively activated and used as the basis for distinguishing between groups (Van Knippenberg et al., 2004). Salience moderates the relationship between demographic diversity and bias, such that highly salient characteristics are more likely to be used to form subgroups, and thus increase the group's tendency towards intergroup bias (Van Knippenberg 
et al., 2004). As we elaborate next, the COVID-19 pandemic creates a social context that heightens the salience of age as a diversity characteristic in the workplace, thereby increasing the risk of bias and creating the opportunity for differential impact.

Public health messaging is one feature of the pandemic that has increased the salience of age in organizations by increasing its cognitive accessibility. The distinct age focus of government health communications throughout the pandemic draws attention to age differences between individuals, making age more easily accessed and retrieved as a diversity characteristic. For example, the Center for Disease Control and Prevention (CDC) website warns that the risk for severe illness from COVID-19 increases with age, stating "people in their 50s are at higher risk for severe illness than people in their 40s. Similarly, people in their 60 s or 70 s are, in general, at higher risk for severe illness than people in their 50 s." Although the emphasis on age is important for public health and safety, a side effect is that it also brings age front of mind when interacting with diverse individuals, increasing the risk of bias and discrimination for those working in age diverse workplaces.

In addition to the emphasis of age in public health messages, the way the risks have, at times, been communicated has been insufficiently nuanced and often over generalised. Older peoples' higher vulnerability to the virus and the more extreme isolation requirements for people over 70 have become inappropriately generalised to all older people and often confound vulnerability with frailty in the public discourse (Hosking et al., 2020). Responding to the increasing ageism in relation to COVID-19 health risks, the Australian Age Discrimination Commissioner stated that “" people over 60” is a very broad group, spanning well over four decades. The needs, health outcomes and characteristics of a typical 60 -yearold are likely to be very different than those of someone in their 80s or older" (Australian Human Rights Commission, 2020). Ayalon et al. (2020) argued that overuse of very broad age categories when reporting risks, and failure to discuss the intersectionality of age with 
other health conditions as a factor that intensifies the risk for those over 50, increases the propensity towards ageism and should be avoided. Older people are already feeling the impact of this overgeneralisation. For example, one respondent to the National Seniors Australia survey, which was released in July 2020, stated "We are being prejudiced. Yes we are at risk, however there needs to be a balance whereby this prejudice does not permeate into the future" (Hosking et al., 2020, p. 29). Although this prejudice often occurs outside the workplace, organisations should be mindful of the way they translate health warnings into health and safety measures in the workplace. Overgeneralising the risk from those of the elderly to all older workers may increase the salience of an individual's age and make age a more cognitively accessible diversity characteristic upon which subgrouping can occur, creating divides between older and younger employees.

The reporting of the pandemic in the popular media also adds to the increased salience of age in the workplace. The markedly negative age and generational focus of popular media's coverage of the pandemic has been widely recognised (Rudolph et al., 2020; Rudolph \& Zacher, 2020). News headlines have described millennials as selfish for ignoring social distancing rules, while others have admonished baby-boomers for hoarding groceries and other essentials. These articles draw on generational stereotypes to unfairly portion blame for COVID-19 outcomes to both old and young individuals. In doing so, they have a polarising effect by reinforcing perceived differences between age diverse people as individuals seek to distance themselves from the perceived bad behaviour of other generations. Although the media's focus has been predominantly focused on non-work topics, negative generational stereotypes and attitudes easily translate to the workplace and can influence bias and discrimination at work, which tends to particularly affect older workers (North, 2019). Consequently, the media contributes to a more negative social climate 
that heightens the risk of hostility and conflict between age diverse team members. This situation has ramifications for knowledge sharing and the overall effectiveness of teams.

The global economic crisis resulting from the pandemic creates additional risk of biased and discriminatory behaviour towards older workers. Studies of past recessions have found that heightened unemployment creates the perception of intensified competition for jobs. This causes a shift towards preserving opportunities for mainstream or prototypical workers, while those who are perceived as part of the out-group (e.g. older workers, women, minorities) are considered less deserving of employment (Vassilopoulou et al., 2019). Vassilopoulou et al. (2019) point to this competition as a source of hostility and resentment towards "the other", who are viewed as taking jobs, resources and opportunities from those considered economically valuable. A pre-pandemic survey found that this was already a concern, with $40 \%$ of respondents aged $18-44$ agreeing with the statement "I feel it is important to retire on time to make way for the next generation" (Andrei et al., 2019). As in previous recessions, these negative attitudes towards older workers are likely to intensify in the aftermath of COVID-19 and limit older workers' access to opportunities, such as promotions, job security, challenging tasks, and training and development opportunities. Simultaneously, many older workers will be forced to keep working due to diminished retirement and superannuation funds. As a result, many mature workers are likely to find themselves trapped in unwelcoming workplaces, undervalued, yet financially unable to retire.

The economic ramifications of the pandemic will also unduly affect older people via changed organizational priorities. Historically, during times of austerity, diversity and inclusion policies and programs have been considered non-essential luxuries that are discontinued to prioritise core functions (Vassilopoulou et al., 2019). This leaves vulnerable those who are reliant on diversity and inclusion policies for access to opportunities. Older workers may be particularly at risk in this situation because age diversity has received 
relatively little industry attention or investment until recently. Therefore, without age-related diversity and inclusion principles guiding organizational decisions, overt age discrimination is more likely. Historically, analysis of unemployment rates during the Great Recession found workers over age 62 and over were the least likely to become reemployed after losing their jobs (Johnson \& Butrica, 2012). Thus, older workers are more vulnerable to institutionalised bias when organizations de-prioritize diversity and inclusion during an economic downturn.

The above arguments paint a picture in which age-based social categorization processes and negative age stereotyping are likely to be enhanced during the pandemic, alongside a climate of increased competition for scarce jobs and reduced protections. We know from existing research that such social and organizational contexts are likely to increase bias towards mature workers and impair the functioning of age-diverse teams. However, whilst we expect these negative effects in many industries, there is a crucial caveat to our perspective. In fact, in industries, such as healthcare, where many mature workers have returned from retirement to support overwhelmed hospitals, the opposite might be true. Here, organizational and societal recognition of the knowledge and skills that older workers have contributed, as well as the personal risk they have taken in returning to work, might help to negate negative social categorization processes. Further, for frontline workers, age might be less salient or meaningful than other diversity characteristics, such as industry or occupation. In the context of heightened health risks and the non-compliance of the general public (e.g., refusal to wear masks), more meaningful similarities may emerge among frontline workers, unifying workers in these industries regardless of age. Further, the extreme work conditions and challenging goals for teams in such industries may further foster group cohesion, unity, and positive intergenerational contact opportunities among age diverse essential workers.

\section{Future research and practice opportunities}


The pandemic creates ample opportunity for research into age bias and its implications for mature workers and age diverse teams. First considering individual and team processes in organizations, we recommend that researchers test our assertion that the pandemic is associated with increased salience of age in the workplace. From this perspective, examining organizations' communication of COVID-19 risks and the implementation of protective health and safety policies could provide insight into the ways in which organizations can either reinforce or erode negative age stereotypes that have been popularised in the media. Further, if the pandemic has in fact increased the salience of age, it is important to assess any impact on work processes in age diverse teams, for example, those working in technology-dependent or physically isolated team environments. The competitive climate that might arise from the pandemic's impact on the economy has the potential to foster a culture of hostility and knowledge hiding, which diminishes the value of age diverse teams. Nevertheless, the pandemic has also created an opportunity to identify organizational strategies that foster positive intergenerational relationships and a culture of knowledge sharing, which will be critical for positive organisational outcomes and economic recovery.

Taking a broader perspective and examining industry differences would be important and interesting. Established research has found that age stereotypes and bias manifest differently across industries (Posthuma \& Campion, 2009). It would be interesting whether the varied impact of the pandemic across industries causes a shift in the influence of stereotypes. For example, do the extreme work conditions in the healthcare industry attenuate the polarising effects of the media and counterbalance negative attitudes typically seen during economic crises? Additionally, it would be valuable to understand the extent to which a competitive job climate varies across industries, and the associated impact on the quality of interactions and collaboration between age diverse team members. We predict that levels of 
bias towards older workers will be greater in industries that have seen the greatest job losses, such as tourism and retail.

There are also research opportunities at the national level. Given the pre-pandemic difficulties that older workers faced in securing employment, some governments are investigating the introduction of dedicated employment support programs for mature workers. For example, the Australian government will introduce a new program to assist older workers re-entering the workforce in the recovery phase of the pandemic (Australian Human Rights Commission, 2020). Evaluating these strategies and comparing them with those embarked on in other countries, will provide insights into opportunities to minimize age discrimination and boost longer term mature worker participation. Also, monitoring the outcomes of individuals and organizations who participate in these government initiatives will enable researchers to continue to develop insights into successful ageing at work.

From a practice perspective, psychologists can play a key role in addressing age bias at all levels, from the individual-level (as identified in Rudolph et al., 2020) to the nationallevel. For the purpose of this article, we focus our discussion on organizational practices. First, it will be important to monitor the social dynamics amongst age diverse team members throughout the pandemic and recovery, and to respond quickly to signs of conflict. Team leaders are ideally placed to address team-level social categorization processes due to their daily contact with their team. Person-focused leader behaviours (e.g., individualized consideration, providing support) will be particularly valuable for minimizing social categorization and fostering a unified team identity (Homan et al., 2020). Second, we advise practitioners to maintain diversity and inclusion programs, despite the financial pressure that might exist to disband them. The long-term benefits of increased innovation, creativity and productivity of age diversity will be critical to long-term global economic recovery. At a 
minimum, practitioners should ensure that decision makers are aware of potential biased attitudes, and approach decisions with inclusivity in mind.

\section{Conclusion}

The pandemic has created a myriad of challenges for workers of all ages, but mature workers are likely to be particularly vulnerable to its effects. Rudolph's et al. (2020) differential susceptibility and impact framework would benefit from expansion to consider social psychology and diversity perspectives. Although age bias may present more subtly than other pandemic-related phenomena, the ramifications can be broad, negatively impacting, individuals, teams, and organizations. The age-focused rhetoric outside the workplace, coupled with increasingly volatile economic environment, increases the likelihood of bias and hence conflict between age diverse individuals in organizations. Industrial and organisational psychology researchers and practitioners are well placed to support organizations navigating these challenges to ensure workplaces are age inclusive despite the challenging context we find ourselves in. 


\section{References}

Andrei, D., Parker, S. K., Constantin, A., Baird, M., Iles, L., Petery, G., Zoszak, L., Williams, A., \& Chen, S. (2019). Maximising potential: Findings from the Mature Workers in Organisations Survey (MWOS) (Industry Report).

Australian Human Rights Commission. (2020). Ageism and COVID-19. https://humanrights.gov.au/about/news/ageism-and-covid-19

Center for Disease Control and Prevention. (2020). Older Adults. https://www.cdc.gov/coronavirus/2019-ncov/need-extra-precautions/older-adults.html

Homan, A. C., Gündemir, S., Buengeler, C., \& van Kleef, G. A. (2020). Leading Diversity: Towards a Theory of Functional Leadership in Diverse Teams. Journal of Applied Psychology. https://doi.org/10.1037/ap10000482

Hosking, D., Ee, N., Maccora, J., Ee, N., \& McCallum, J. (2020). COVID-19: Older Australians' life and care during the pandemic (Issue July).

Johnson, R. W., \& Butrica, B. A. (2012). Age Disparities in Unemployment and Reemployment during the Great Recession and Recovery. In Unemployment and Recovery Project (Issue May).

North, M. S. (2019). A gate to understanding “older" workers: Generation, age, tenure, experience. Academy of Management Annals, 13(2), 414-443. https://doi.org/10.5465/annals.2017.0125

Posthuma, R. A., \& Campion, M. A. (2009). Age stereotypes in the workplace: Common stereotypes, moderators, and future research directions. Journal of Management, 35(1), 158-188. https://doi.org/10.1177/0149206308318617

Rudolph, C. W., Allan, B., Clark, M., Hertel, G., Hirschi, A., Kunze, F., Shockley, K., Shoss, 
M., Sonnentag, S., \& Zacher, H. (2020). Pandemics: Implications for Research and Practice in Industrial and Organizational Psychology. Industrial and Organizational Pyschology: Perspectives on Science and Practice.

Rudolph, C. W., \& Zacher, H. (2020). “The COVID-19 Generation”: A Cautionary Note. Work, Aging and Retirement, 6(3), 139-145. https://doi.org/10.1093/workar/waaa009

Tajfel, H., \& Turner, J. C. (1986). An integrative theory of intergroup relations. Psychology of Intergroup Relations, 7-24.

Thatcher, S. M. B., \& Patel, P. C. (2012). Group Faultlines: A Review, Integration, and Guide to Future Research. Journal of Management, 38(4), 969-1009. https://doi.org/10.1177/0149206311426187

Turner, J. C., Hogg, M. A., Oaks, P. J., Reicher, S. D., \& Wetherell, M. S. (1987). Rediscovering the social group: A self-categorization theory. Blackwell.

Van Knippenberg, D., De Dreu, C. K. W., \& Homan, A. C. (2004). Work group diversity and group performance: An integrative model and research agenda. Journal of Applied Psychology, 89(6), 1008-1022. https://doi.org/10.1037/0021-9010.89.6.1008

Vassilopoulou, J., Kyriakidou, O., da Rocha, J. P., Georgiadou, A., \& Mor Barak, M. (2019). International Perspectives on Securing Human and Social Rights and Diversity Gains at Work in the Aftermath of the Global Economic Crisis and in Times of Austerity. European Management Review, 16(4), 837-845. https://doi.org/10.1111/emre.12333 